\title{
Effects of encoding and retrieval on the mechanism of item + context binding
}

\author{
XIAO Xin ${ }^{1}$, DING JinHong ${ }^{1} \&$ GUO ChunYan ${ }^{1 *}$ \\ Beijing Key Laboratory of Learning and Cognition and Department of Psychology, Capital Normal University, Beijing 100089, China
}

Received October 25, 2010; accepted January 21, 2011; published online May 10, 2011

\begin{abstract}
To investigate the neural mechanism of semantic representation and color context binding, we used electroencephalograph time frequency and coherence analyses to reveal local and long-range functional coupling in the encoding and retrieval phases of episodic memory. Fifteen undergraduates participated in the experiment and middle-frequency double-character Chinese words were used as stimuli in two types of study-test tasks (context recall and context recognition tests). Significant differences between item+context and item in the encoding phase were observed at the electrodes in the frontal region at $600-800 \mathrm{~ms}$ by time frequency analysis. Further differences were observed at $800 \mathrm{~ms}$ by independent component analysis: the frontal component and the coherent component of the triangle phase locking structure among the prefrontal, right parietal and left parietal-occipital regions. In the retrieval phase, differences between item+context and item were found on the electrodes at the central parietal and parietal-occipital regions at $400 \mathrm{~ms}$ by time frequency analysis, on the parietal-occipital component at $800 \mathrm{~ms}$ by independent component analysis and on the coherence component of the anterior right hemisphere and parietal-occipital regions at $1400 \mathrm{~ms}$. In conclusion, the different effects of encoding and retrieval processing on 'binding' are reflected by the differing extents that brain regions engaged in cognitive operations. In the retrieval phase in particular, activities of the parietal-occipital region were specifically associated with 'binding', and coherence between frontal and temporal-parietal regions is a common brain activity in episodic memory.
\end{abstract}

encoding, retrieval, binding, item, context, frontal and parietal-occipital coherence

Citation: Xiao X, Ding J H, Guo C Y. Effects of encoding and retrieval on the mechanism of item+context binding. Chinese Sci Bull, 2011, 56: 1787-1798, doi: $10.1007 / \mathrm{s} 11434-011-4501-4$

Complex object processing requires dynamic collaboration among multiple brain structures. Observations from patient and brain imaging studies suggest that encoding information into long-term memory depends on frontal and parietal lobes [1]. Memorizing central content and episodic context of an event may be functionally dissociated and rely on different anatomical regions. Schizophrenia is linked to a deficit in retrieving contextual information and in binding the different components of memory together [2]. An important goal of memory research is to determine how perceptual experiences are transformed into new memories. It is insufficient to encode content and context independently, and the

*Corresponding author (email: guocy@ hotmail.com) brain has to build 'binding' between them to form episodic memory of a certain event. However, few studies have addressed how different brain regions interact during the formation of new memories $[1,3,4]$.

According to dynamic approaches of brain function, synchronous oscillations reflect an index of sharing information among spatially separate brain regions. This method emphasizes the interaction of brain areas in cognition activities [5]. Klimesch et al. [6] reported greater electroencephalograph (EEG) coherence across brain areas during tasks that required information integration such as perception, sensorimotor integration and working memory. EEG coherence reflects the basic neural mechanism of functional connectivity across multiple neural structures for perception, intentional binding, sensory awareness and even subjective 
self-perception [1].

Synchronization of the theta band was reported to reflect the encoding of new information and episodic memory and a greater degree of synchronization (neural synchronization triggered by the activity of two or more neurons or neural ensembles oscillating with a repeated sequence of relative phase angles) for remembered words than for forgotten words [7]. By contrast, other studies reported that significant theta synchronization was only found in later remembered and correctly recognized target words, rather than in distract and non-remembered targets. Using the Remember/Know paradigm, Opitz [8] found that the temporal dynamics of theta synchronization were related to the particular conscious experiences associated with memory retrieval. In their recognition test, subjects indicated whether they consciously recollected the events in which a word was earlier presented (Remembered) or whether they recognized the events on the basis of familiarity (Known) in the absence of recollection. The authors concluded that an early EEG synchronization in the theta band predicted 'know' while later EEG synchronization predicted 'remember'. Crespo-Garcia et al. [9] reported that the frontal and parietal regions promoted successful encoding, and that coherent activities between the frontal and parietal areas were correlated with successful recall and recognition of words. Furthermore, successful encoding of the semantic characters of a word stimulus required the sharing of information among the ventral stream association areas, visual word form areas and left frontal regions $[1,10]$.

Retrieval of time-spatial information is an important property of episodic memory. The hippocampus, prefrontal lobe and amygdala are connected in episodic memory. As the central part of retrieval, the hippocampus combines different elements of experiences [11]. Discriminating old or new in a recognition task is located in the frontal and parietal areas of the brain, which are important for memory in the retrieval phase [3]. EEG coherence or phase synchronization of neural oscillation reflects the fundamental mechanism by which the brain associates across neural spaces. Increases in local synchrony over frontal areas have been observed during the encoding of words that were later correctly identified as old word in an old/new recognition test. However, there is no evidence that long-range coherence between frontal and parietal regions can separate old/new item or true/false recognition. Naghavi et al. [12] reported the common frontal-parietal activity in terms of processes related to integration of distributed representations in episodic retrieval. fMRI and PET studies with a variety of designs and experimental conditions have also revealed the involvement of the consistent neural signals of frontal and parietal regions in episodic memory retrieval.

In this study we examined the differences between item and item+context, and the functional connectivity of different brain regions in the encoding and retrieval phases of episodic memory. We recorded EEG activities when sub- jects learned or recognized words in different font colors. In the context recall test we classified encoding trials on the basis of two levels of later memory performance: trials in which the word+color association was successfully encoded (WC) and trials in which successful word recognition occurred but without encoding of the associated color (WO trials). According to whether subjects could report the color of words they learnt, we separated the stimuli into different conditions. In the context recognition test we divided stimuli into word+color retrieval (hit) and word retrieval (false alarm) depending on whether subjects could recognize the color. In addition, we assumed that investigation of the interaction of different brain regions would explain the neural mechanism of binding. Thus, we adopted BEAST for time-frequency, coherence and independent component analysis (ICA) $[1,13,14]$.

\section{Materials and methods}

\subsection{Participants}

Fifteen undergraduate students ( 7 male, mean age $=19.7$ years) from Capital Normal University participated in the experiment. All participants were right-handed and had normal or corrected-to-normal vision, with Chinese as their first language.

\subsection{Stimuli}

The stimuli were 600 two-character Chinese words with a low frequency from 20-80 occurrences/million. The colors of words included: Blue (RGB-33, 9, 206), Green (RGB-25, 148, 50), Red (RGB-165, 23, 25) and Yellow (RGB-182, $182,54)$. Colors were randomly arranged and grouped to form 90 words for each color. To reduce interruptions from high correlations of word and color, we excluded easily imagined words such as 'green grass'.

\subsection{Procedure}

(i) Context recall test. First, participants performed a four-block context recall test. In the study/encoding phase, we presented 40 word-color pairs with a 2000 -ms duration and an interstimulus interval (ISI) of $1000 \pm 100 \mathrm{~ms}$. Subjects were told that the word was the item and the color was the corresponding context. Subjects were asked to remember 40 pairs. After the study phase there was a short distraction phase in which a 3-digit number was presented, and subjects were asked to subtract backwards by 3 and gave vocal reports for $60 \mathrm{~s}$. In the test/retrieval phase we presented 80 white probe words, half old and half new. The duration and ISI of the words were identical within the study phase. Subjects were asked to indicate whether the probe word was new or old, and they should report the color of old words that they had learned (Figure 1). 


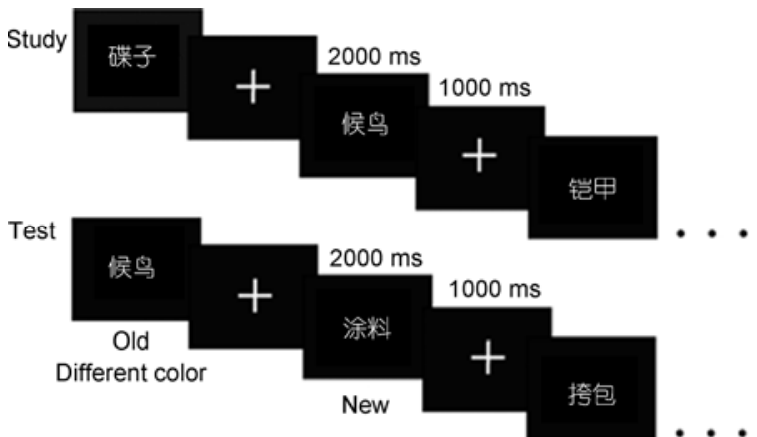

Figure 1 Context recall test procedure. Half of the words were old and half were new in the test phase. Subjects indicated whether the word was new or old. After an 'old' response, subjects spoke the color of word.

(ii) Context recognition test. After the context recall test, 4 study-test blocks of the context recognition test were performed. In the study/encoding phase, we presented 50 word-color pairs. Subjects were told that the word was the item and that the color was the corresponding context, and they were asked to remember the 50 pairs. After the study phase there was a short distraction task for $60 \mathrm{~s}$. In the test/retrieval phase we presented 70 probe word-color pairs, 50 old and 20 new. When compared with the study phase, 30 word-color pairs were old words with different colors, while 20 pairs were old words with the same color. Subjects were required to discriminate whether the word was old or new, and for the old word they should indicate whether the color was the same. Old words with the same color required a key press, while old words with a different color or a new word required a different key press (Figure 2).

\subsection{EEG recordings and data analysis}

We used the study-test paradigm to investigate the different effects of encoding and retrieval on the neural mechanism of binding. In the context recall experiment, we investigated the effect of encoding on binding and only analyzed the EEGs of the encoding phase. According to whether subjects

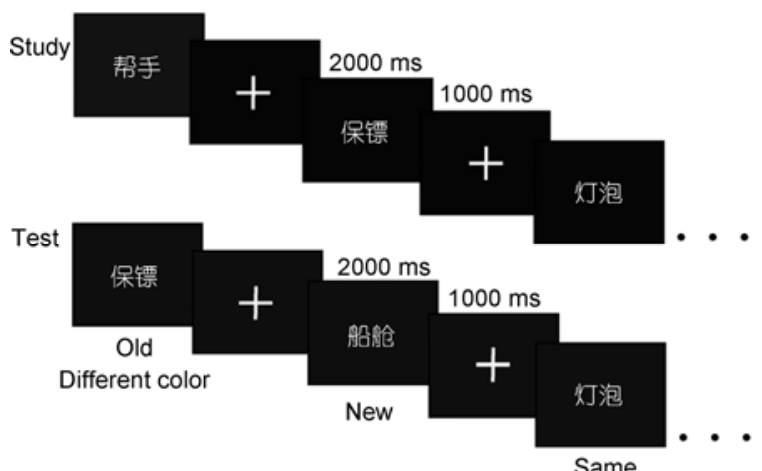

Figure 2 Context recognition test procedure. Subjects indicated whether the word-color pairs were the same between the study and test phases. Same pairs required one key press, while an old word with different color pairs or with new word pairs required a different key press. correctly reported the color of 'old' words, we separated stimuli into word+color and word conditions in the encoding phase. In the context recognition experiment we investigated the effects of retrieval on binding and only analyzed the EEGs of the retrieval phase. After a 'hit' response (i.e., correct response to the same word+color pairs) we classified this type of stimuli as a successful retrieval of word and color. By contrast, after a 'false alarm' response (i.e., incorrect responses to the old words with different colors) we classified this type of stimuli as a retrieval of word only because the subjects failed to recognize the color.

EEG recordings were recorded from $62 \mathrm{Ag} / \mathrm{AgCl}$ electrodes embedded in a cap of ESI-64-channel electrophysiology recording analysis system. The electrodes were located according to an extension of the international 10-20 system. Vertical electrooculography (EOG) was recorded by the electrodes placed directly above and below the supra-orbital of left eye, and horizontal EOG was monitored via a bipolar montage at the outer canthi of both eyes. Reference electrodes were located on left and right mastoids. The ground electrode was between Fpz and Fz. The impedances between scalp and electrodes were kept below $8 \mathrm{k} \Omega$. EEG signals were filtered with a band-pass of $0.05-100 \mathrm{~Hz}$ and sampled at a rate of $500 \mathrm{~Hz}$.

First, we removed artifacts from the EOG channels and filtered EEG signals with a high-pass of $0.5 \mathrm{~Hz}$ zero phases in the off-line analysis. Second, we processed data with BEAST v2.0, which is based on Matlab [1,13]. The function of BEAST was to extract a different frequency from the EEG data and to perform ICA and coherent analysis according to local and long-range neural synchronized activities. Spatial ICA was used to decompose the time-frequency (TF) and coherence data into spatially correlated components. ICA was used to reduce the quantity of EEG data into spatially independent components. ICA provides a 'blind' solution to separate statistically independent signals, without the constraint of orthogonalizing the factors intrinsic to principal component analysis. Coherence analysis measures the similarity of EEG signals in the frequency domain. Numerous studies have demonstrated that coherence analysis is an effective tool to investigate functional connectivity between cognitive processes of different cortical regions. EEG coherence is the synchronism of various frequencies in different brain regions and indicates whether identical EEG frequency signals recorded from different brain areas are independent or dependent. Coherence analysis has a crucial role in understanding the functional connectivity in spatial relations within the whole brain.

BEAST data processing consists of three stages: (i) signal preprocessing: EEG data were baseline corrected by subtracting the mean amplitude of the -500 to $0 \mathrm{~ms}$ window from the entire epoch, and the 'induced' theta activity remained; (ii) TF decomposition: All the epochs were Morlet wavelet-transformed between 4 and $8 \mathrm{~Hz}$, generating mean $\mathrm{TF}$ information for each condition, at each electrode, for 
each subject; (iii) coherence: Because of the quantity of computation, coherence was calculated for a set of 20/81 electrodes (FPZ, AF3, AF4, F7, F8, FCZ, FC3, FC4, C1, C2, T7, T8, CP3, CP4, PZ, P7, P8, PO3, PO4, and OZ) [1,13], and these electrodes provided full scalp sites with at least 6 $\mathrm{cm}$ between any given electrode pairs.

\section{Results}

\subsection{Behavioral analyses}

A $2 \times 3$ repeated-measure ANOVA of accuracy rate consisting of tasks (context recall and context recognition) $\times$ memory conditions (word, word+color, new word) was conducted (Table 1). There were significant main effects for tasks $[F(1,14)=24.60, P<0.01]$ and memory conditions $[F(2,28)=105.67, P<0.01]$, and significant interactions among the tasks $\times$ memory conditions $[F(2,28)=21.79, P<$ $0.01]$. The main effect analysis revealed that in the word+color condition there was a significant difference between context recall and context recognition $[F(1,14)=$ 51.26, $P<0.01]$. There were significant differences among the word, word+color and new word conditions in the context recall test $[F(2,28)=114.38, P<0.01]$ and in the context recognition test $[F(2,28)=79.96, P<0.01]$. In addition, in post hoc analyses there were significant differences for word+color to word, new word to word and new word to word+color $[P s<0.01]$.

We performed a $2 \times 3$ repeated-measure ANOVA of reaction time for tasks (context recall and context recognition) $\times$ memory conditions (word, word+color, new word). There was a significant main effect of the tasks $[F(2,28)=28.79$, $P<0.01]$, but no main effect for the memory condition and no significant interactions among the tasks $\times$ memory conditions. Post hoc analysis reveals that there were significant differences for word+color to word, new word to word and new word to word+color $[P s<0.01]$.

\subsection{TF and coherence analyses}

(i) Local theta activity in the encoding phase: electrode-wise analysis. We compared different brain activities between word+color and word in the encoding phase, and determined the mean spectral power in the theta band between 0 and $1400 \mathrm{~ms}$ poststimulus interpolated across the scalp for each of the two conditions. We chose the 5 electrodes that corresponded to the greatest significance for each comparison (Figure 3(a) and (b)), and determined the frontal brain activities for the word+color condition (Figure 3(a), left) and right parietal-occipital brain activities for the word condition (Figure 3(a), right). Differences between the WC and WO trials were mainly observed at the left prefrontal, frontal-parietal and parietal electrodes (Figure 3(b)). The time courses of theta band activity for three representative electrodes (FP2, AF4 and AF7) are shown in Figure 3(c). Mean spectral power (in $z$ scores) across time ( $x$-axis, from $0-1400 \mathrm{~ms}$ poststimulus) and frequency ( $y$-axis, from $4-8$ $\mathrm{Hz}$ ) is plotted for each electrode for the WC (left) and WO (right) conditions. We found variation in the times of theta band activities for the WC and WO conditions. According to the comparison of mean power spectrum for each condition in the $0-1400 \mathrm{~ms}$ window, we obtained the five electrodes that showed the greatest differences (Figure 3(a) and (b)). The criteria for selecting the representative electrodes were a significant difference between conditions in the single electrode comparison and having one of the greatest differences (Figure 3(b)). In Figure 3(d), TF significance maps show $P$ values for TF pixels reaching a $P<0.05$ threshold for the comparison WC $>$ WO. A theta increase was initially seen in all conditions (Figure 3(c)). However, this response was more sustained in the WC trials in the 200-800 ms window, and less extended for WO in the 200-400 ms time window. Thus, significant differences correlating to successful encoding mainly occurred late in the epoch of 600-800 ms at 5-6 Hz (Figure 3(d)).

(ii) Local theta activity in encoding: ICA. According to the ICA of the word+color and word conditions in the encoding phase, results showed that the frontal (component 1) and parietal-occipital (component 2) components accounted for the greatest percentage of variance ( $2.89 \%$ for component 1 and $6.04 \%$ for component 2; Figure 4(a)). The TF profile of the activations of component 1 showed a robust and sustained poststimulus theta response for $\mathrm{WC}$ and $\mathrm{WO}$ in the 0-400 ms window (Figure 4(b), left), and the theta response receded for WO in the 400-800 ms window at 6-8 $\mathrm{Hz}$ (Figure 4(b), left down). There was a significant difference between WC and WO at $800 \mathrm{~ms}$ between $7-8 \mathrm{~Hz}$ (Figure 4(c), left). For component 2, the TF profile of the activations showed a robust and sustained poststimulus theta response for $\mathrm{WC}$ and $\mathrm{WO}$ in the $0-200 \mathrm{~ms}$ window at 6-8 $\mathrm{Hz}$ and in the 200-400 ms window at 4-6 Hz (Figure 4(b), right). However, there were no significant differences between WC and WO (Figure 4(c), right). Other components also accounted for a high percentage of variance, but showed no significant differences between WC $>$ WO, which was the same as for that in component 2 .

Table 1 Accuracy rate and mean reaction time of context recall and context recognition tests (standard errors in brackets)

\begin{tabular}{|c|c|c|c|c|c|c|}
\hline & \multicolumn{2}{|c|}{ Word } & \multicolumn{2}{|c|}{ Word+color } & \multicolumn{2}{|c|}{ New word } \\
\hline & $\mathrm{AP}(\%)$ & RT (ms) & $\mathrm{AP}(\%)$ & $\mathrm{RT}(\mathrm{ms})$ & $\mathrm{AP}(\%)$ & $\mathrm{RT}(\mathrm{ms})$ \\
\hline Context recall & $27.72(0.03)$ & $1077(86)$ & $53.78(0.04)$ & $952(58)$ & $90.33(0.02)$ & $810(27)$ \\
\hline Context recognition & $25.40(0.03)$ & $1163(55)$ & $72.20(0.03)$ & $971(32)$ & $92.73(0.02)$ & $888(28)$ \\
\hline
\end{tabular}


(a)
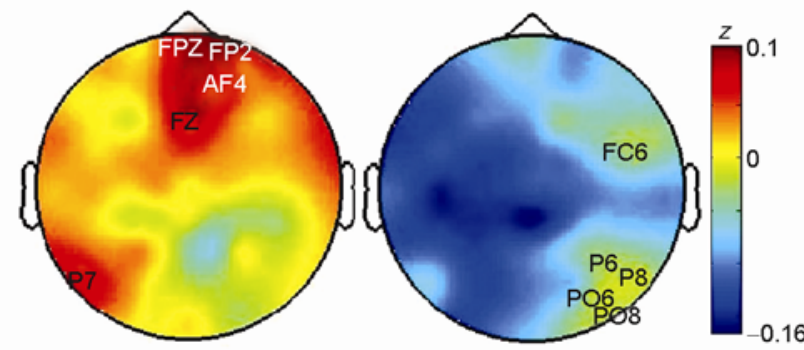

FP2

(c)
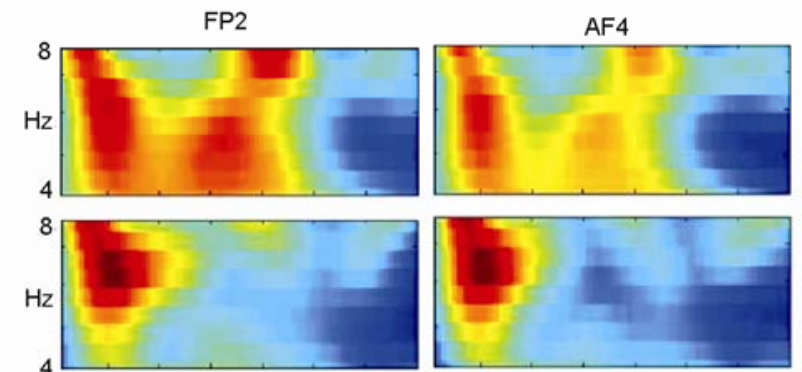

(b)
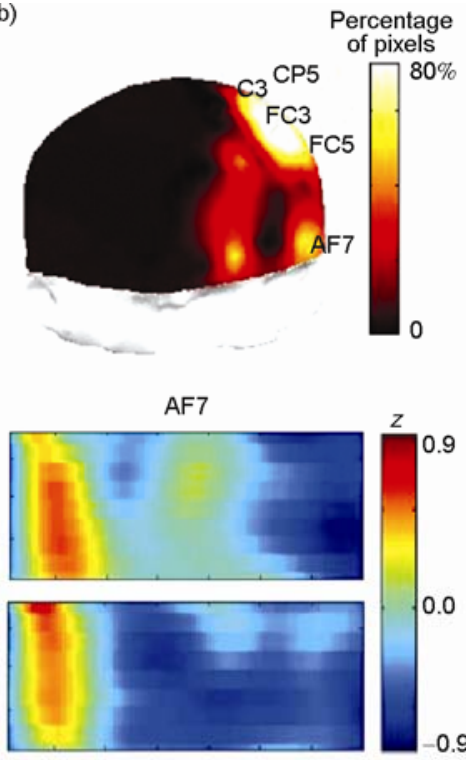

WO
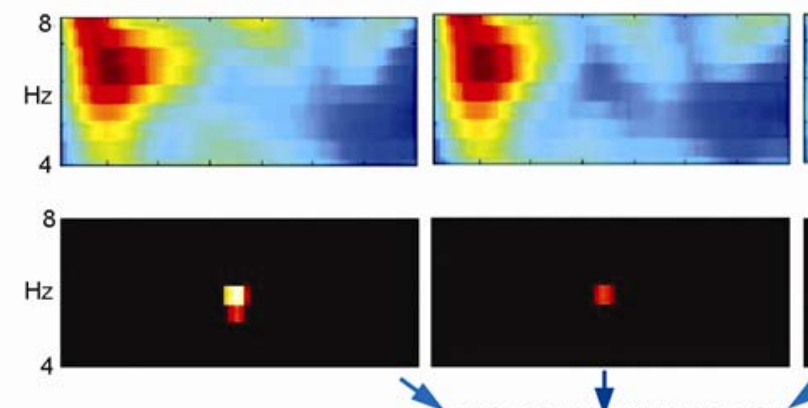

200400600800100012001400

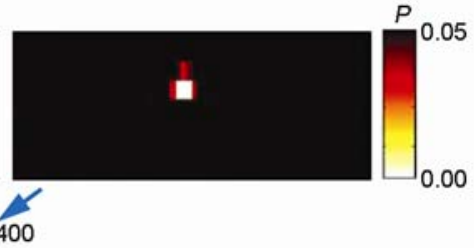

Figure 3 Time-frequency analysis of local frontal theta activities. (a) Mean theta power across the whole epoch for WC (left) and WO (right). (b) Brain regions showing significant differences in theta power for the comparison WC $>$ WO. Scale refers to percentage of pixels reaching $P<0.05$ significance. (c) Mean time-frequency profiles of theta-band activity for three representative electrodes. (d) Regions of time-frequency space showing significant differences for the comparisons.

(iii) Long-range theta activity in the encoding phase: ICA. We used 20 of the 64 electrodes to form 190 electrode pairs to compute coherence. Consistent with previous studies $[1,13]$, to reduce the 190 electrode pairings to a manageable number of components, we employed spatial ICA with 10 components. We plotted the seven electrodes with the highest weights (grey lines) on top of the total component weights for each electrode interpolated across the scalp. We selected 8 electrode pairs with high weights by permutation and combination of 7 electrodes. We chose standards from 10 components, including the components showing significant differences between the different conditions, brain areas based on previous studies and those showing the greatest variation $[1,13]$. According to the coherent ICA of the word+color and word conditions in the encoding phase, the results show that one component accounts for the greatest percentage of variance $(14.8 \%$, Figure $5(\mathrm{a}))$. All 8 of the electrode pairings showed phase locking within a triangle network encompassing prefrontal, right parietal and left parietal-occipital electrode sites: FPZ-PO3, FPZ-PO4, FPZ-P8, AF3-P8, AF4-PO3, AF4-OZ, AF4-PO4 and AF4-P8 (Figure 5(a)). The results show mean 'corrected' coherence across TF space. Coherence began at stimulus onset in the theta $(5-7 \mathrm{~Hz})$ and alpha $(8-12 \mathrm{~Hz})$ bands for the WC and WO conditions (Figure 5(b)). The results of coherence show a robust and sustained poststimulus theta response for $\mathrm{WC}$ at $0-500 \mathrm{~ms}$ in the theta and alpha bands, and after $500 \mathrm{~ms}$ in the alpha band (Figure 5(b)). Furthermore, coherent activities were weak for WO at 400-900 ms (Figure 5(b)). There was a significant difference between $\mathrm{WC}$ and $\mathrm{WO}$ at $800 \mathrm{~ms}$ between 9 and $11 \mathrm{~Hz}$ (Figure 5(c)).

(iv) Local theta activity in the retrieval phase: electrode-wise analysis. We determined the different brain activities among the word+color, word and new word conditions in the retrieval phase, and presented the mean spectral power in the theta band between 0 and $1400 \mathrm{~ms}$. The parietal-occipital brain activities were greater for the WC condition (Figure 6(a), left), prefrontal brain activities were greater for the WO condition (Figure 6(a), middle) and parietal-occipital brain activities were greater for the NEW condition (Figure 6(a), right). Differences among WC, WO and NEW trials were mainly observed at the right hemisphere electrodes (Figure 6(b)), showing central-parietal at the right hemisphere and parietal-occipital regions for WC $>$ WO (Figure 6(b), left), prefrontal at the right hemisphere and frontal-temporal regions for $\mathrm{WC}<\mathrm{WO}$ (Figure 6(b), middle), and temporal at right hemisphere and central regions for WC $>$ NEW (Figure 6(b), right). The time courses of theta-band activity for the 3 representative electrodes (CP4, FT8 and C6) are shown in Figure 6(c). There were 
(a)
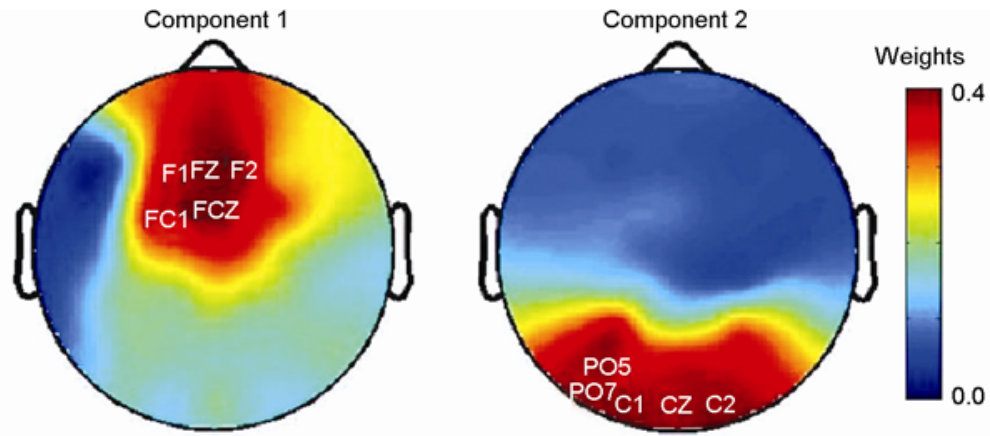

(b)
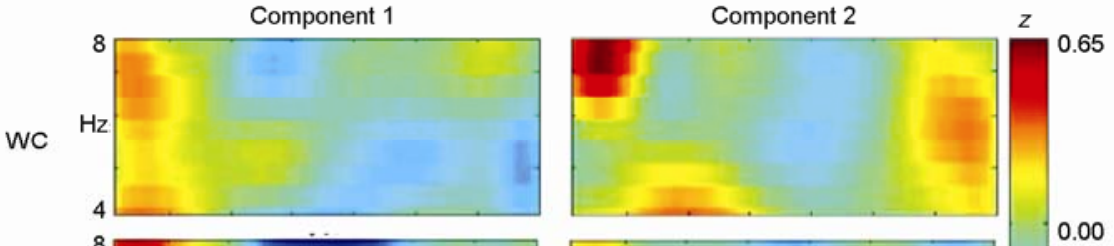

wo
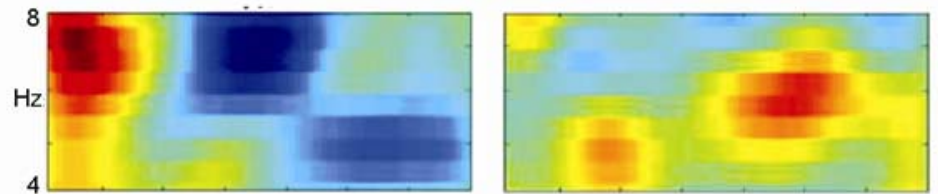

(c)

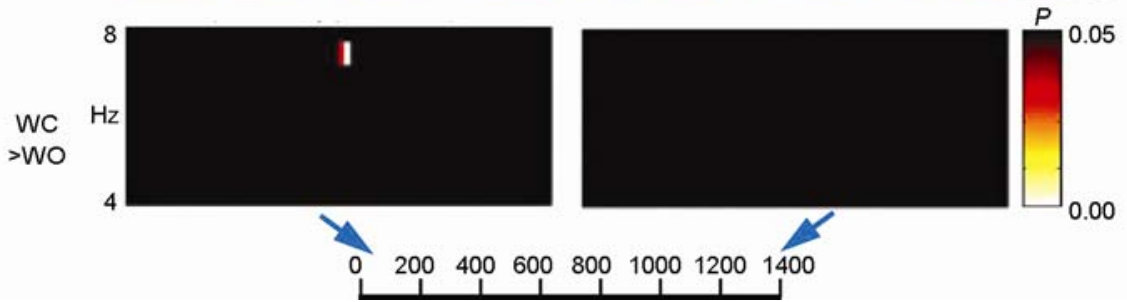

Figure 4 ICA of local theta activity. (a) Scalp distribution of ICA components 1 and 2. The scale refers to the ICA weight. (b) Mean time-frequency for each component for each condition. (c) Areas of time-frequency space showing significant differences for the comparison WC > WO.

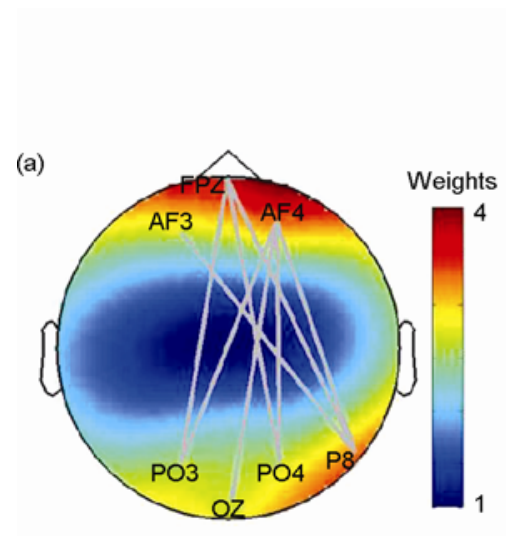

(b)

WC
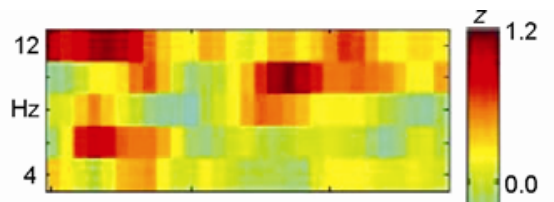

WO

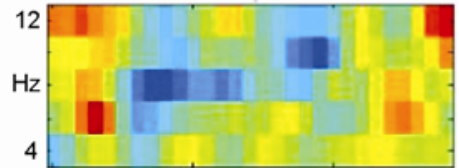

(c)

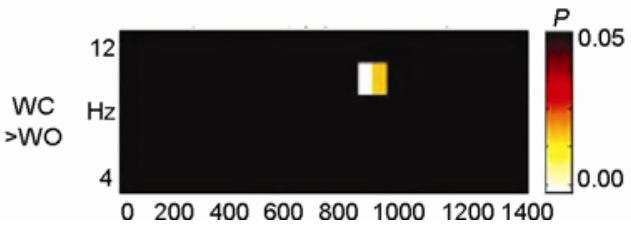

Figure 5 ICA of long-range theta coherence. (a) The component showing significant differences between conditions. The 8 electrode pairings are those with the highest weights. The color represents the weights of ICA. (b) Mean corrected coherence across time-frequency space for the component. (c) Areas of time-frequency space that show a high coherence for the comparison $\mathrm{WC}>\mathrm{WO}$.

variations in the theta band activities for $\mathrm{WC}$, WO and NEW conditions with time from the onset of stimulus.

The theta response of WC was more persistent and continuous than WO in the epoch of 200-600 ms (Figure 6(c)). There were significant differences among the comparisons
$\mathrm{WC}>\mathrm{WO}, \mathrm{WC}<\mathrm{WO}$, and $\mathrm{WC}>\mathrm{NEW}$ at $400 \mathrm{~ms}$ between 5 and $7 \mathrm{~Hz}$ (Figure 6(d), left), at $1200 \mathrm{~ms}$ between 4 and $5 \mathrm{~Hz}$ (Figure 6(d), middle) and at $400 \mathrm{~ms}$ between 6 and $7 \mathrm{~Hz}$ (Figure 6(d), right).

(v) Local theta activity in the retrieval phase: ICA. Ac- 
(a)

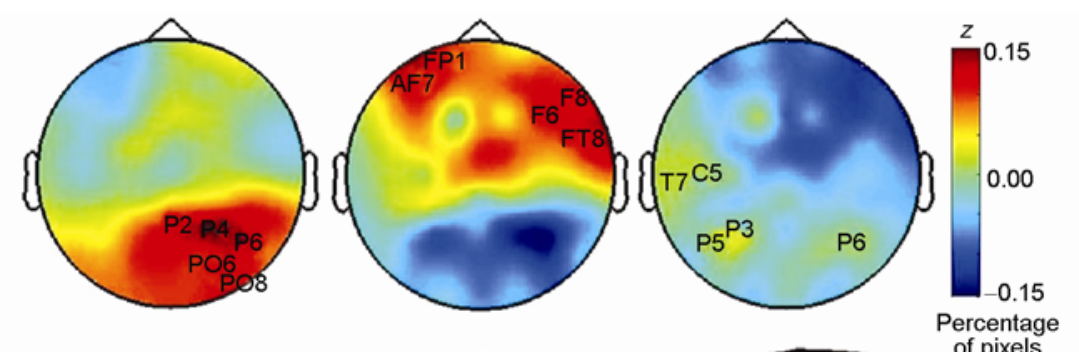

(b)

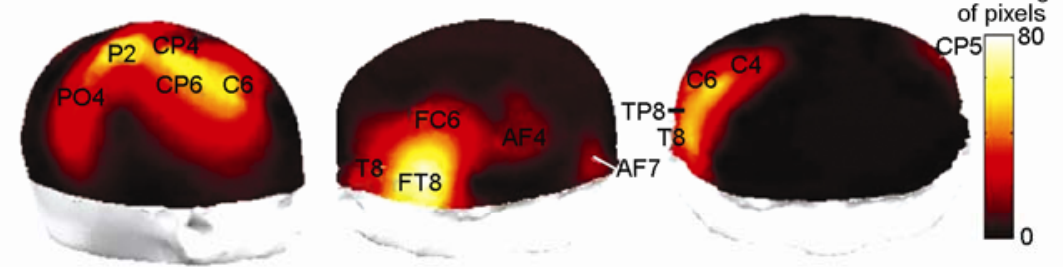

(c)
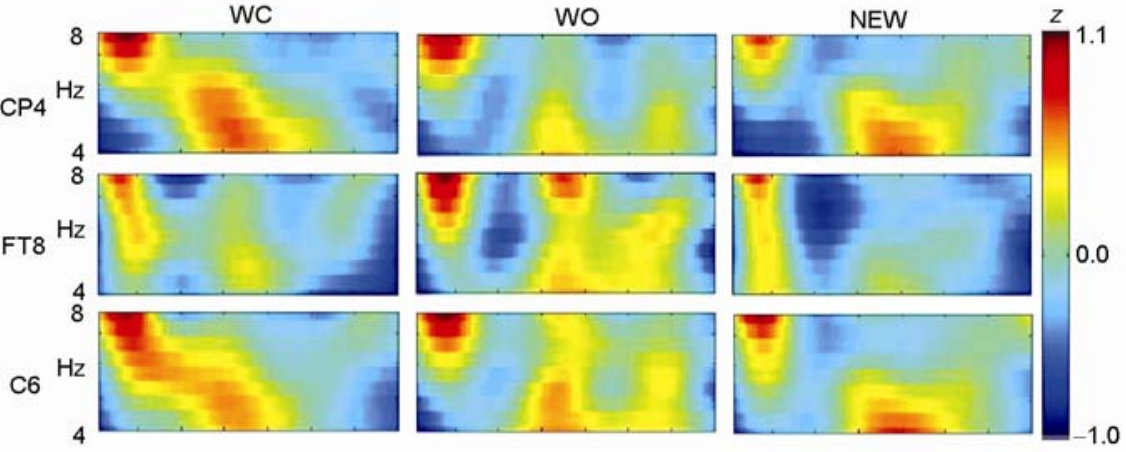

(d)
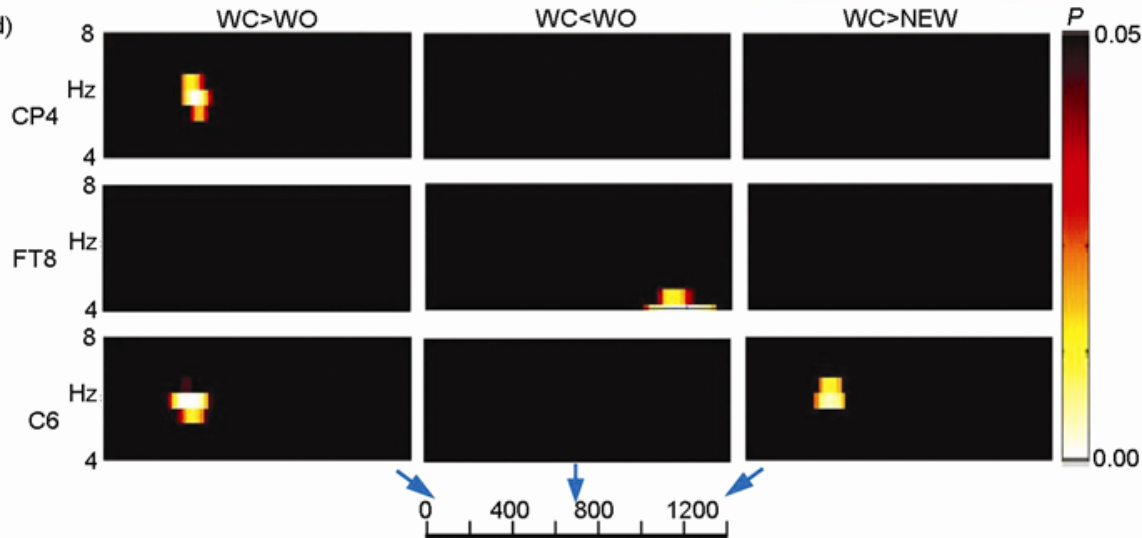

Figure 6 Time-frequency analysis of local frontal theta activities. (a) Mean theta power across the epoch for WC (left), WO (middle) and NEW (right). (b) Brain regions showing significant differences in theta power for the comparisons WC $>$ WO (left), WC $<$ WO (middle) and WC $>$ NEW (right). (c) Mean time-frequency profile of theta-band activity for three representative frontal electrodes. (d) Regions of TF space showing significant differences for the comparisons.

cording to the ICA of WC, WO and NEW conditions in the retrieval phase, the parietal-occipital (component 1, Figure 7(a), left) and frontal (component 2, Figure 7(a), right) components accounted for the greatest percentage of variance $(4.04 \%$ for component 1 and $2.59 \%$ for component 2 ). The TF profile of the activations of component 2 shows a robust and sustained poststimulus theta response for $\mathrm{WC}$, WO and NEW in the $0-300 \mathrm{~ms}$ window. Further, activation of component 1 exhibited a theta response for $\mathrm{WC}$, WO and NEW beginning at $300 \mathrm{~ms}$ and continuing in the 300-600 $\mathrm{ms}$ and $800-1000 \mathrm{~ms}$ windows (Figure 7(b)). There were significant differences between the comparisons WC>WO and $\mathrm{WC}<\mathrm{WO}$ at $800 \mathrm{~ms}$ between 6 and $8 \mathrm{~Hz}$ (Figure 7(c), component 1 , left) and in the 800-1000 ms window at 4-5 $\mathrm{Hz}$ (Figure 7(c), component 2, middle). However, there were no significant differences between $\mathrm{WC}>\mathrm{NEW}$ for components 1 and 2 (Figure 7(c)).

(vi) Long-range theta activity in the retrieval phase: ICA. Results show that the component accounting for the greatest percentage of variance $(7.3 \%)$ exhibited significant differences between WC and WO in the retrieval phase (Figure 8(a)). All eight electrode pairings with the greatest weights of this component showed phase locking between the frontal and parietal-occipital electrode sites of the right 
(a)

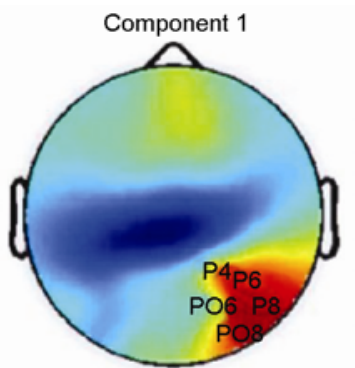

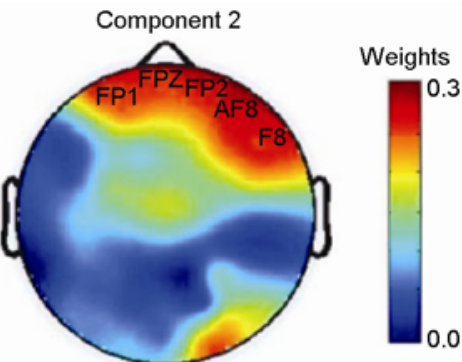
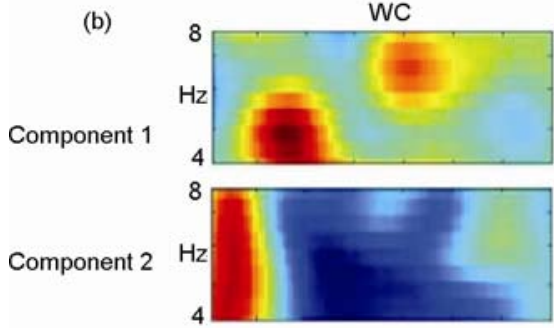

(c)

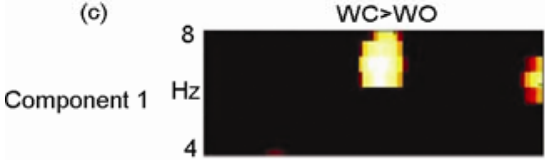

Component 2
WO
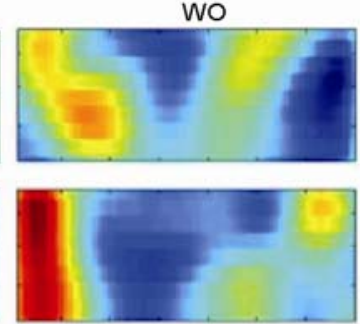

WC $<$ WO
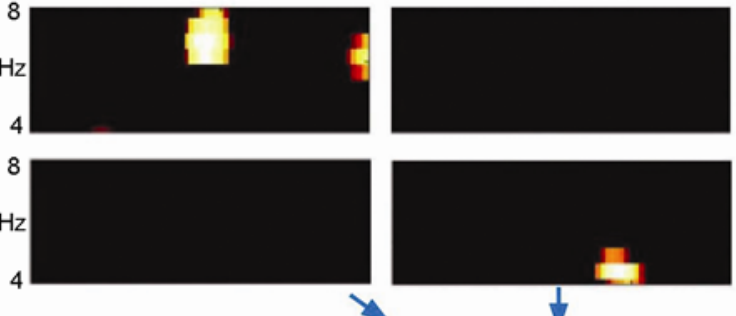

200400600800100012001400
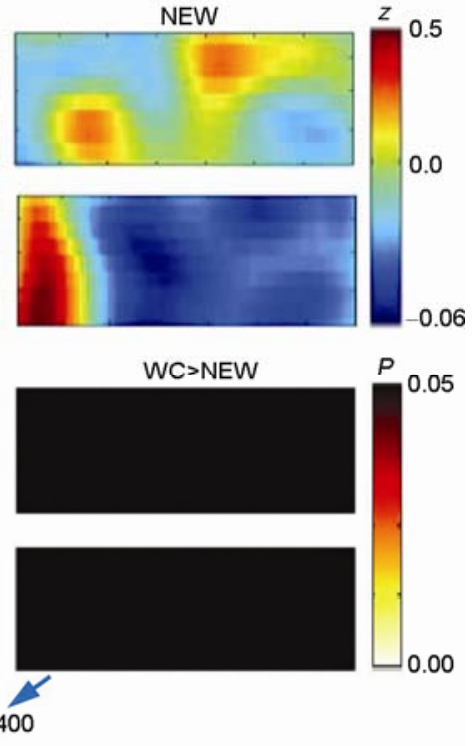

Figure 7 ICA of local theta activities. (a) Scalp distribution of ICA components 1 and 2. (b) Mean component activations for each component for each condition. (c) Areas of time-frequency space showing significant differences for the comparisons across the different conditions.

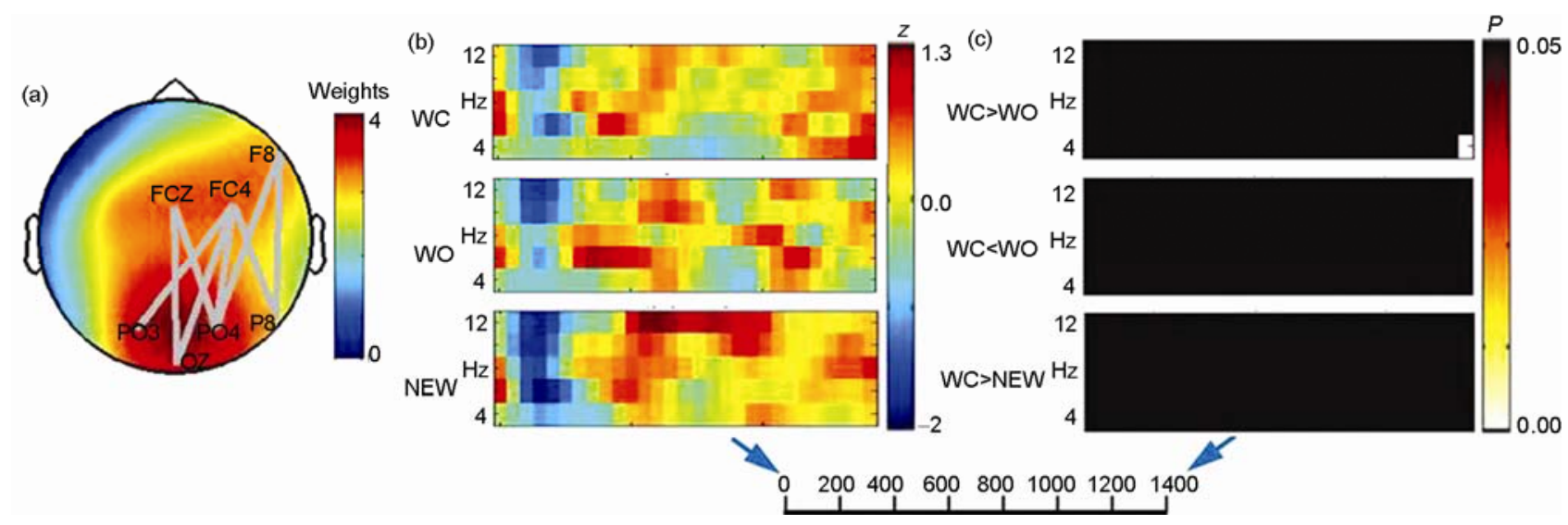

Figure 8 ICA of long-range theta coherence. (a) The component showing significant differences between conditions. (b) Mean corrected coherence across time-frequency space for the component. (c) Areas of time-frequency space showing a high coherence for the comparisons across different conditions.

hemisphere: FCZ-OZ, FCZ-PO4, FC4-PO3, FC4-OZ, FC4PO4, FC4-P6, F8-PO4 and F8-P6. According to the coherent ICA, there was a robust and sustained theta coherent response for WC and WO from 300-1400 ms (Figure 8(b)). This component showed significant differences in coherence for the comparison WC>WO peaking at $1400 \mathrm{~ms}$ between 4 and $5 \mathrm{~Hz}$ (Figure 8(c)).

\section{Discussion}

According to our behavioral analyses, we found that for the NEW condition, reaction time was shorter and accuracy rate was higher in both the context recall test and the context recognition test, which is consistent with other studies [15]. One explanation is that the old-new effect reflects retrieving 
an item or ignoring the context. In addition, a shorter reaction time and a higher accuracy rate were observed by comparing item+context with item in the two tasks. In the context recall task, subjects were asked to make a 'remember' response after recalling the context and a 'know' response when forgetting the context. In other words, there was an extra retrieval attempt before the response. In the context recognition task the results are consistent with other studies reporting that the hit rates were higher for matched item+context responses (word+color condition), while false alarm was lower for unmatched item+context responses (word condition) [8]. Moreover, the longer reaction time for item condition reflects the false recognition process $[16,17]$.

\subsection{The synchronized neural activities of item+context binding in the encoding phase}

The increases of theta band power in the epoch of 600-700 $\mathrm{ms}$ for the comparison item+context (word+color) with item (word) reflect specific activities for the encoding of item+context binding (Figure 3), which is consistent with previous studies [1]. Theta synchronized activities were reported to increase in the encoding phase within the frontal regions in which were later identified as old word in old/new recognition. This continuous frontal theta response predicted the successful encoding of words, which reflected the encoding of new information, attention demand, task difficulty, increased cognition demand in episodic memory and rehearsal or elaboration in verbal working memory $[10,18]$. According to local theta ICA, the frontal component showed a significant difference between item+context and item at $800 \mathrm{~ms}$ (Figure 4). An fMRI study reported that activation in the right inferior frontal gyrus (BA 44, corresponding to the place of our electrode F8) predicted successful encoding of word+color [19]. An ERP study compared 'remember $>$ know' responses and found significant differences in the right anterior frontal region in the 400-600 ms time window [8]. One explanation is that a 'top-down' control mechanism located in the prefrontal lobe contributes to deciding what perceptual information should be processed and form episodic memory trace in the episodic encoding phase $[4,9,20]$. In each phase of the perceptual processing, a 'control' mechanism in the frontal area selects perceptual representations and rejects other information synchronously, reflecting the different neural signals between successful and unsuccessful encoding. Forming long-term memory is the function of the medial temporal lobe, where the perceptual information is finally accessed, and where the episodic details are integrated $[11,21]$.

\subsection{The synchronized neural activities of comparing item+context binding with item in the retrieval phase}

In the present study, we compared item $>$ item+context in the late window (after $1000 \mathrm{~ms}$ ) located in the frontal sites, and found that the theta activities in local frontal regions were relative to the retrieval of an item (Figure 6). Using ICA, we examined component 2 in the prefrontal areas (Figure 7) compared with false memory. We demonstrated that the frontal component was engaged in judgment processing in the retrieval phase. In case where participants made a false alarm response (i.e., in the test phase subjects failed to recognize the old words that appeared in a different color from the study phase), we considered that they did not retrieve the context information. Previous behavioral and neural imaging studies have revealed the different neural mechanism of true and false memory. For example, falsely recognized events that participants did not experience have been predicted by the activities in left prefrontal lobe because source monitor processing is absent when making false recognition [16,20]. Word materials have also been used to determine EEG activities between true and false memories, and the ERP waveform was shown to be more negative when comparing false alarms with hits in the frontal and parietal lobe areas [17]. Furthermore, when participants thought they answered correctly with a high confidence but in fact made a wrong response, the increased activities in the frontal-parietal region were found related to 'familiarity' and not 'recollection' [22].

In addition, the theta response in the parietal-occipital region is connected with the retrieval of item+context (Figure 6). The difference between item+context and item is located in the central parietal region at the $400 \mathrm{~ms}$ window, which is similar to comparing item+context with new item. Recent studies showed that the theta power of 'remember' was greater in the 450-650 ms window, which was related to the increase of consciousness or attention. Increased theta power reflects the complicated circuits of the hippocampus-cortex in the 'remember' response and the activities of the perirhinal cortex and medial dorsal nucleus of the thalamus in the 'know' response [8,23]. Moreover, ICA confirmed that the right parietal-occipital component was significantly different between item+context and item in the $800 \mathrm{~ms}$ window (Figure 7, component 1). Cycowicz et al. [24] discovered the episodic memory effect (EM) or old-new effect, which indicates that the difference between correct recognition of old items and correct rejection of new items reaches a maximum in the parietal-occipital region. Context processing in the learning phase is located in parietal-occipital brain regions, which reflects the searching or retrieval of context (color). EM effects peaked at the 800-1000 ms window, which reflects recovering the context of picture in the context retrieval task. Parietal activities have also reported to increase when comparing recognition of old items (hits) with correct rejection of new items, false recognition of new items (false alarm) and forgetting old items (miss) in episodic retrieval [25]. In summary, this increasing of parietal activities exists in different materials, response conditions and tasks. Bilateral and central-parietal are related to remembering experience i.e., when recollection 
of event and details of context occurs, activation of parietal increase. There are two explanations for the EM effect: searching or retrieval item+context and retrieval 'binding' information. The partial-occipital region is important to retrieval context and binding information. Opitz [8] reported that one strategy was imaging item+context to decide whether it was matched or unmatched in context retrieval; when unmatched it was hard to recall. Binding information contributes to making correct responses, while item and context appear at the same time in the test rather than presenting item independent or item with different context.

\subsection{The coherent analysis of binding activities in the encoding and retrieval phases}

We observed long-range theta coherent activities between the frontal and parietal-occipital electrodes (Figure 5). A similar study observed significant different coherent component between CB1 (P7 in this study) and AF3 [1]. In addition, other study reported the function of the ventral association region in semantic retrieval [26]. One possibility is that neurons exhibit sustained firing at the theta rhythm phase in the left frontal structures in item encoding, which elicits a phase-locked response from neurons in the left parietal-occipital association regions. This activity may form a correlated neural integration during item encoding, which contributes to item successful encoding i.e., it is more likely for the increasing coherent activities between two brain areas to generate a 'remember' response than for increasing coherent activities in a local brain region [27].

Consistent with a recent study [1], we confirmed that sustained frontal-parietal coherence occurred in the lower alpha band $(9-11 \mathrm{~Hz})$ at approximately $800 \mathrm{~ms}$, which predicted the binding activities of item+context according to ICA between item+context and item in the encoding phase. Previous ERP studies reported that the right frontal and parietal-occipital activities in the late epoch of encoding reflected the neural integration of the two regions and predicted the 'remember' response of words [28]. A working memory study also reported coherent activities between the frontal and parietal-occipital regions, which are related to word encoding in successful recall and recognition [29]. Current models of working memory assume that parietal-occipital perceptual and association areas exhibit sustained activity through connection with the prefrontal area during short-term storage. Frontal lobe regions act as an intentional 'pointer' that maintains representations in the parietal-occipital area online and proceeds semantic elaboration. The synchronized activities of the theta band has an important role in the interaction between the anterior and parietal-occipital regions during working memory maintenance and elaboration, suggesting the possibility of entering into long-term memory $[6,10]$. In the present study, ICA of coherence in the retrieval phase showed a significant difference in the frontal-occipital component that was centered at $1400 \mathrm{~ms}$ in the lower theta band when comparing item + context with item (Figure 8). A previous neural imaging study demonstrated that the frontal and parietal lobes are activated when we recall experienced events, and true recognition requires the functional connectivity of those regions [13]. The models of working memory indicate that the representations of sensory information in the parietal region are activated by executive control mechanisms of the frontal lobe, and this mechanism is engaged in reactivating details of sensory representations.

Some studies have investigated frontal and parietal-occipital coherent activities in the gamma band in the encoding phase, where they assumed that the activities reflected the functional 'binding' among temporal-spatial information in scenes, representations of inter- and intra- semantic memory and the intentions of subjects [30]. In the retrieval phase, frontal-occipital coherence has been shown to be related to functional 'binding' of the representations elicited by the visual scenes that were similar to the previous episode [5]. The connections between the prefrontal and parietal-occipital regions (including parietal, parietal-occipital and occipital areas) reflected the interaction between central executive and storage. When neurons in the prefrontal region integrated different features in encoding and retrieval processing, long-range connection has a crucial role in the working memory $[28,31,32]$. According to anatomical studies, there is a high connectivity between the frontal and parietal areas. For example, when the parietal cortex of the stump-tailed monkey was cooled, activities of neurons in the prefrontal decreased correspondingly [12]. These results illustrate the functional interaction of two brain regions, and prove that the functions of the parietal and prefrontal regions were for storage and maintenance or updating, separately.

\subsection{The neural mechanisms of binding activities be- tween the encoding and retrieval phases}

From the time course analyses of the encoding and retrieval phases, we discovered that the difference of ICA of TF appeared early while the difference of coherent analysis appeared late when comparing item+context with item. In the encoding phase the difference in the frontal component was centered at $800 \mathrm{~ms}$, and the difference in the frontal-parietal coherent component was centered at 800-1000 ms. We considered that the frontal component in the theta band at $800 \mathrm{~ms}$ was specifically associated with successful encoding of item+context binding [1]. According to the time course, coherent activities between the frontal and parietal-occipital regions appeared soon after the stimulus onset, but the difference between item+context $>$ item appeared later. From this point of view, we conclude that the functional connection is a dynamic activity. When one region is insufficient for the current demand of task, the coherent activities are inclined to decrease. In the retrieval phase the 
difference in the parietal-occipital component was centered at 700-900 ms, while the frontal-parietal coherent component was centered at $1400 \mathrm{~ms}$. Recognition is a 'reactivation' process which includes retrieval attempt i.e., when subjects recall context information, if parietal brain region does not satisfy the demand of task then there is no successful retrieval of context. Coherent activities will decrease after several failures of retrieval when subjects are not capable of finishing the task. We assumed that the parietal-occipital component in the theta band at 700-900 ms is specifically associated with successful retrieval of item+ context binding [24].

One explanation of binding activities among different brain regions is that a tripartite system exists with cooperative interactions between the PFC, hippocampus or medial temporal lobe area (MTL) and the parietal-occipital cortex [9,11,21,33-35]. The top-down control of PFC provides selection, modification and elaboration of the representations in the parietal-occipital cortex within the encoding process, and provides memory search and information selection from the parietal-occipital cortex in the retrieval process. The PFC may indirectly interact with the MTL, such that representations are controlled by the PFC and stored via the MTL in the parietal-occipital cortex. Moreover, the prefrontal cortex and the perirhinal and entorhinal cortices are strongly reciprocally connected, which leads to a direct interaction. A possible functional explanation of the direct interaction is the role in the integration and combination of spatial and temporal context of events. In addition, the PFC is capable of integrating the outcomes of diverse cognitive operations and controlling processing according to spatial and temporal context demands. In other words, direct access from the PFC to the MTL may provide the contextual information to transform into a spatial-temporal specific episodic memory trace.

\section{Conclusions}

In summary, the frontal component is a crucial neural mechanism for binding in the encoding phase when comparing item+context with item. However, the success of context retrieval depends on the parietal-occipital brain region. In addition, we found a common frontal and parietal-occipital network in the encoding and retrieval phases, and determined activities in this network were dynamically connected. However, if one region does not satisfy the current task demands, coherent activities were inclined to decrease. We confirmed that the different effects of encoding and retrieval processing on 'binding' are reflected by the different extents of brain regions engaged in cognitive operations. In the retrieval phase in particular, activities of the parietal-occipital region that were specifically associated with 'binding', and coherence between the frontal and temporal-parietal regions were common brain activities in epi- sodic memory.

The authors express their great thanks to Christopher Summerfield for support with analysis with BEAST, and thank Tian Tian for assistance with editing the manuscript. This work was supported by the National Natural Science Foundation of China (30870760), the Key Foundation of Beijing Municipal Commission of Education (KZ201010028029) and the Chinese Ministry of Education (20101108110004) to Guo Chunyan. The work was also supported by the National Natural Science Foundation of China (30970891) and the Doctoral Fund of Ministry of Education of China (20091108110001) to Ding Jinhong.

1 Summerfield C, Mangels J A. Coherent theta-band EEG activity predicts item-context binding during encoding. NeuroImage, 2005, 24: 692-703

2 Waters F A V, Maybery M T, et al. Context memory and binding in schizophrenia. Schizophr Res, 2004, 68: 119-125

3 Babiloni C, Babiloni F, Carducci F. Human cortical EEG rhythms during long-term episodic memory task: A high-resolution EEG study of the HERA model. NeuroImage, 2004, 21: 1576-1584

4 Summerfield C, Greene M, Wager T, et al. Neocortical connectivity during episodic memory formation. PLoS Biol, 2006, 4: 855-864

5 Engel A K, Fries P, Singer W. Dynamic predictions: Oscillations and synchrony in top-down processing. Nat Rev Neurosci, 2001, 2: 704-716

6 Klimesch W, Freunberger R, Sauseng P. Oscillatory mechanisms of process binding in memory. Neurosci Biobehav Rev, 2010, 34: 1002-1014

7 Klimesch W. EEG alpha and theta oscillations reflect cognitive and memory performance: A review and analysis. Brain Res Rev, 1999, 29: 169-195

8 Opitz B. Context-dependent repetition effects on recognition memory. Brain Cogn, 2010, 73: 110-118

9 Crespo-Garcia M, Cantero J L, Pomyalov A, et al. Functional neural networks underlying semantic encoding of associative memories. NeuroImage, 2010, 50: 1258-1270

10 Sauseng P, Griesmayr B, Freunberger R, et al. Control mechanisms in working memory: A possible function of EEG theta oscillations. Neurosci Biobehav Rev, 2010, 34: 1015-1022

11 Gottlieba L J, Uncapherb M R, Rugg M D. Dissociation of the neural correlates of visual and auditory contextual encoding. Neuropsychologia, 2010, 48: 137-144

12 Naghavi H R, Nyberg L. Common fronto-parietal activity in attention, memory, and consciousness: Shared demands on integration? Conscious Cogn, 2005, 14: 390-425

13 Summerfield C, Mangels J A. Functional coupling between frontal and parietal lobes during recognition memory. Neuroreport, 2005, 16: 117-122

14 Summerfield C, Mangels J A. Dissociable neural mechanisms for encoding predictable and unpredictable events. J Cogn Neurosci, 2006, 18: 1120-1132

15 Guo C Y, Duan L, Li W, et al. Distinguishing source memory and item memory: Brain potentials at encoding and retrieval. Brain Res, 2006, 1118: 142-154

16 Kubota Y, Toichi M, Shimizua M, et al. Prefrontal hemodynamic activity predicts false memory-A near-infrared spectroscopy study. NeuroImage, 2006, 4: 1783-1789

17 Walla P, Endl W, Lindinger G, et al. False recognition in a verbal memory task: An event-related potential study. Brain Res Cogn Brain Res, 2000, 9: 41-44

18 Liu Z M, Guo C Y. Interactions between working memory and selective attention. Chinese Sci Bull, 2007, 52: 2811-2819

19 Ranganath C, Yonelinas A P, Cohen M X, et al. Dissociable correlates of recollection and familiarity within the medial temporal lobes. Neuropsychologia, 2004, 42: 2-13

20 Guo C Y, Chen W J, Tian T, et al. Orientation to learning context modulates retrieval processing for unrecognized words. Chinese Sci Bull, 2010, 55: 1831-1838 
21 Verguts T, Notebaert W. Adaptation by binding: A learning account of cognitive control. Trends Cogn Sci, 2009, 13: 252-257

22 Kim H, Cabeza R. Trusting our memories: Dissociating the neural correlates of confidence in veridical versus illusory memories. $\mathrm{J}$ Neurosci, 2007, 27: 12190-12197

23 Klimesch W, Doppelmayr M, Yonelinas A. Theta synchronization during episodic retrieval: Neural correlates of conscious awareness. Cogn Brain Res, 2001, 12: 33-38

24 Cycowicz Y M, Friedman D, Snodgrass J G. Remembering the color of objects: An ERP investigation of source memory. Cereb Cortex, 2001, 11: 322-334

25 Wagner A D, Shannon B J, Kahn I, et al. Parietal lobe contributions to episodic memory retrieval. Trends Cogn Sci, 2005, 9: 445-453

26 Thompson-Schill S L. Neuroimaging studies of semantic memory: Inferring 'how' from 'where'. Neuropsychologia, 2003, 41: 280-292

27 Weiss S, Rappelsberger P. Long-range EEG synchronization during word encoding correlates with successful memory performance. Cogn Brain Res, 2000, 9: 299-312

28 Mangels J A, Picton T W, Craik F I M. Attention and successful epi- sodic encoding: An event-related potential study. Cogn Brain Res, 2001, 1: 77-95

29 Sauseng P, Klimesch W, Doppelmayr M, et al. Theta coupling in the human electroencephalogram during a working memory task. Neurosci Lett, 2004, 354: 123-126

30 Nyhus E, Curran T. Functional role of gamma and theta oscillations in episodic memory. Neurosci Biobehav Rev, 2010, 34: 1023-1035

31 Zimmer H, Mecklinger A, Lindenberger U. Handbook of Binding and Memory: Perspectives from Cognitive. Oxford: Oxford University Press, 2006

32 Summerfield C, Egner T, Greene M, et al. Predictive codes for forthcoming perception in the frontal cortex. Science, 2006, 314: 1311-1314

33 Liu R, Guo C Y, Jiang Y. An event-related potential study of working memory in children. Chinese Sci Bull, 2006, 51: 1467-1475

34 Meng Y F, Guo C Y. ERP dissociation and connection between implicit and explicit memory. Chinese Sci Bull, 2007, 52: 2945-2953

35 Slotnick S D. Does the hippocampus mediate objective binding or subjective remembering? NeuroImage, 2010, 49: 1769-1776

Open Access This article is distributed under the terms of the Creative Commons Attribution License which permits any use, distribution, and reproduction in any medium, provided the original author(s) and source are credited. 